\title{
Correlation of Fibroblast Growth Factor 23 with Markers of Inflammation and Endothelial Dysfunction in End-Stage Renal Disease and Type 2 Diabetes Patients on Peritoneal Dialysis
}

\author{
Ruchi Singh, Sudha P Chennasamudram, Sudip Sheth and Tetyana L Vasylyeva* \\ Texas Tech University Health Sciences Center, Amarillo, Texas, USA \\ "Corresponding author: Tetyana L Vasylyeva, Texas Tech Health Sciences Center, Pediatrics, 1400 S. Coulter, Amarillo, TX 79106, USA, Tel: 1-806-351-3796; \\ Fax: 1-806-354-5791; E-mail: tetyana.vasylyeva@ttuhsc.edu
}

Rec date: Apr 16, 2014, Acc date: May 16, 2014, Pub date: May 20, 2014

Copyright: (c) 2014 Singh R, et al. This is an open-access article distributed under the terms of the Creative Commons Attribution License, which permits unrestricted use, distribution, and reproduction in any medium, provided the original author and source are credited.

\begin{abstract}
Objective: This study was design to evaluate the correlation between FGF-23 and other cardiovascular risk factors (markers of endothelial dysfunction and inflammation) in ESRD patients with T2DM on PD.

Method: Sixty serum samples collected (four times, at weeks $0,8,10$, and 18) from 15 patients with Type 2 Diabetes (T2DM) and ESRD on PD were analysed to study the relationship between the levels of FGF-23 and markers of endothelial dysfunction and inflammation. ELISA kits were used to quantitate Endothelin-1 (ET-1), soluble vascular adhesion molecule, Plasminogen Activator Inhibitor-1 (PAI-1), FGF-23, C-reactive protein, Interleukin-6 (IL-6), Tumor Necrosis Factor Alpha (TNF- $\alpha$ ) and cluster of differentiation 146 (CD 146). The association between FGF-23, mineral metabolites, and markers of inflammation were analyzed using Spearman correlations. Linear regression models were used to examine the univariate and multivariate adjusted associations between FGF-23 as primary exposure and individual inflammatory markers [including IL-6, PAI-1 and CD146] as the dependent variables. Multivariate analyses were adjusted for factors associated with mineral metabolism such as serum phosphate, CaxPO4 product, and LDL concentration. Two-sided $\mathrm{p}$ values $<0.05$ were considered statistically significant.
\end{abstract}

Result: FGF-23 was positively correlated with phosphate $(r=0.57 ; p<0.0001)$ and CaxPO4 product $(r=0.61$; $p<0.0001)$. FGF-23 showed the strongest correlation with IL-6 ( $r=0.32 ; p<0.05)$, PAI-1 $(r=0.21 ; p<0.05)$ and CD 146 $(r=0.29 ; p<0.05)$. In univariate and multivariate regression analysis, FGF-23 was significantly associated with IL-6, PAI-1, and CD 146. These results were qualitatively unchanged in the model that was further adjusted for CaxPO4 product, serum phosphate, and LDL.

Conclusion: Our results indicate that FGF-23 impacts the cardiovascular health of T2DM patients on PD through mechanisms, which are independent from phosphate levels and linked directly to inflammation and endothelial dysfunction.

Keywords: Fibroblast growth factor-23; End-stage renal disease; Peritoneal dialysis; Inflammatory markers; Type 2 diabetes; Endothelial dysfunction; Mineral metabolites

\section{Introduction}

Distorted bone and mineral metabolism are considered as risk factors for cardiovascular mortality in patients with End-Stage Renal Disease (ESRD). Fibroblast Growth Factor-23 (FGF-23), produced in the bone, is a strong predictor of adverse cardiovascular events in ESRD patients [1]. A strong correlation exists between FGF-23 level and mortality in this patient [2-5]. FGF-23 is considered as a unique risk marker for the cardiovascular mortality in Chronic Kidney Disease (CKD) patients [6]. The FGF-23 concentration remains unchanged throughout the day in CKD patients [7]. Increased Soluble Parathyroid Hormone (S-PTH) and FGF-23 levels are associated with the progression of vascular calcification in Peritoneal Dialysis (PD) patients [8].

Gutierrez et al. reported that increased levels of FGF-23 are linked with a noticeable increase in mortality and sickness in CKD patients,
ESRD patients, and kidney transplant recipients [3]. However, the clinical outcome in ESRD patients is also associated with the level of systemic inflammation and endothelial dysfunction. Elevated serum C-reactive protein (CRP) is a strong and more robust predictor of cardiovascular mortality than well-established risk factors such as LDL cholesterol level. The association between elevated CRP and increased mortality is reported in both Hemodialysis (HD) $[9,10]$ and PD $[8,11]$ populations. Several previous experimental, clinical, and interventional studies have documented that endothelial dysfunction; vascular calcification, oxidative stress, and inflammation are central factors in the complex control of mineral metabolism [12]. Patients with diabetes are the most vulnerable due to compromised metabolism [13].

Recent studies demonstrated that vascular disease is an inflammatory condition [14,15]. Cytokines initiate and coordinate inflammatory responses that involve increased hepatic production of acute phase reactants, including CRP, interleukin 6 (IL-6), Tumor Necrosis Factor-alpha (TNF- $\alpha$ ), and cluster of differentiation 146 (CD146) $[10,16]$. Elevated serum levels of pro-inflammatory cytokines 
Citation: $\quad$ Singh R, Chennasamudram SP, Sheth S, Vasylyeva TL (2014) Correlation of Fibroblast Growth Factor 23 with Markers of Inflammation and Endothelial Dysfunction in End-Stage Renal Disease and Type 2 Diabetes Patients on Peritoneal Dialysis. J Diabetes Metab 5: 371. doi:10.4172/2155-6156.1000371

Page 2 of 5

such as CRP and intact Parathyroid Hormone (iPTH) have been linked to increased mortality in dialysis patients [16]. A strong association is observed between inflammation and atherosclerosis in Hemodialysis (HD) patients [3,16]. Inflammatory markers are independent predictors of atherosclerotic plaques in the carotid arteries $[9-12,15]$. These inflammatory markers are strong predictors of subsequent cardiovascular disease and cardiovascular mortality, both in the general population and among patients on dialysis [14] Inflammation is believed to be linked to endothelial dysfunction [14].

The aim of our study was to evaluate the correlation between FGF-23 and other more traditional cardiovascular risk factors (CRP, lipids, and markers of endothelial dysfunction and inflammation) in ESRD patients with T2DM on PD.

\section{Methods}

Sixty serum samples collected four times at weeks $0,8,10$, and 18 from 15 adult subjects between 18- and 65-year-old with T2DM on PD whose serum phosphate levels were above $5.5 \mathrm{mmol} / \mathrm{L}$ were analyzed. Patients were enrolled from the Amarillo Kidney Specialist, LLC dialysis unit after providing informed consent. The study protocol was approved by the Texas Tech University Health Sciences Center Institutional Review Board. Subjects with Parathyroid Hormone (PTH) levels greater than $1,000 \mathrm{mmol} / \mathrm{L}$, calciphylaxis, ora history of hypercalcemia within the past three months were excluded from the study. Whole blood was collected in a vacutainer (Becton Dickinson, Franklin Lake, NJ). After collection of the blood, it was allowed to clot at room temperature for 30 minutes. The clot was removed by centrifugation at $1,000-2,000 \times \mathrm{g}$ for 10 minutes in a refrigerated centrifuge. Serum was transferred to clean polypropylene tubes and 0.5 $\mathrm{ml}$ aliquots were stored at $-20^{\circ} \mathrm{C}$. Serum samples were analyzed for FGF-23, ET-1, PAI-1, soluble Vascular Adhesion Molecule (sVCAM), soluble Intercellular Adhesion Molecule (sICAM), IL-6, Interleukin $1 \beta$ (IL-1 $\beta$ ), TNF- $\alpha$, CRP, and CD146. Endothelial function was evaluated by measurement of flow mediated dilation by Endo-PAT (Itamar Medical, Franklin, MA). In addition, serum was analyzed for albumin, calcium, phosphate, PTH, and lipids by a commercial laboratory (Quest Laboratory, Amarillo, TX).

\section{Serum FGF-23, sVCAM, sICAM, ET-1, PAI-1, CD146 and pro-inflammatory cytokines}

Enzyme-linked Immunosorbent Assays (ELISAs) were used according to manufacturer's instructions (R\&D Systems, Minneapolis, MN) to measure the serum levels of FGF-23, sVCAM, sICAM, ET-1, PAI-1, IL-6, IL-1 $\beta$, TNF- $\alpha$, and CRP. The direct ELISA method was used to measure CD146 levels [17].

\section{Statistical analysis}

ELISA data were analyzed by using 4 parameter logistic equations (Supplementary data). Continuous variables were summarized as mean \pm SEM (Table 1). FGF-23, IL-6, CRP, TNF- $\alpha$, were natural log (ln)-transformed. Associations between FGF-23, mineral metabolites, and markers of inflammation were analyzed using Spearman correlations. Univariate and multivariate regression analyses were conducted to examine the relationships between FGF-23, inflammatory markers (IL-6), markers of endothelial dysfunction (PAI-1, CD146), and mineral metabolites. In these, analyses were limited to the inflammatory markers that were found positive and significantly correlated with FGF-23 by Spearman test.

\begin{tabular}{|c|c|}
\hline \multicolumn{2}{|l|}{ Socio-demographic } \\
\hline Sex & 8 female, 7 male \\
\hline Age (Years) & $56.6 \pm 2.4$ \\
\hline BMI & $34.0 \pm 1.3$ \\
\hline \multicolumn{2}{|l|}{ Anemia } \\
\hline Hematocrit (\%) & $35.49 \pm 0.61$ \\
\hline HCT CALC (HGBX3) & $34.94 \pm 0.62$ \\
\hline Hemoglobin $(\mathrm{g} / \mathrm{dL})$ & $11.68 \pm 0.20$ \\
\hline \multicolumn{2}{|l|}{ Iron management } \\
\hline Iron saturation (\%) & $34.47 \pm 3.51$ \\
\hline Iron & $79.57 \pm 7.78$ \\
\hline $\operatorname{TIBC}(\mu \mathrm{g} / \mathrm{dL})$ & $233.18 \pm 9.05$ \\
\hline UIBC $(\mu \mathrm{g} / \mathrm{dL})$ & $149.67 \pm 12.46$ \\
\hline \multicolumn{2}{|l|}{ Osteodystrophy } \\
\hline Calcium (mg/dL) & $9.11 \pm 0.33$ \\
\hline Phosphorus (mg/dL) & $5.75 \pm 0.48$ \\
\hline Cal×Phos product & $51.56 \pm 4.48$ \\
\hline Alkaline Phosphatase (mg/dL) & $111.13 \pm 20.40$ \\
\hline Calcium corrected & $9.35 \pm 0.32$ \\
\hline Cal $\times$ Phos product corrected & $53.11 \pm 4.68$ \\
\hline \multicolumn{2}{|l|}{ Nutrition and Lipids } \\
\hline Potassium (mg/dL) & $4.49 \pm 0.13$ \\
\hline Albumin (mg/dL) & $3.70 \pm 0.10$ \\
\hline \multicolumn{2}{|l|}{ Adequacy of dialysis and renal function } \\
\hline Creatinine ( $\mu$ moles $/ \mathrm{L}$ ) & $7.44 \pm 0.81$ \\
\hline Blood Urea Nitrogen & $54.73 \pm 3.04$ \\
\hline $\begin{array}{l}\mathrm{Kt} / \mathrm{V} \text { ( } \mathrm{K}=\text { dialyzer clearance of urea, } \mathrm{t}=\text { dialysis } \\
\text { time and } \mathrm{V}=\text { volume of distribution of urea in } \\
\text { the body) }\end{array}$ & $2.47 \pm 0.13$ \\
\hline Residual renal function (mL/Min) & $6.68 \pm 1.36$ \\
\hline Duration of dialysis & $\begin{array}{l}9 \text { patients ( }>6 \text { months) } \\
6 \text { patients (<6 Months) }\end{array}$ \\
\hline Dialysis regimen & $\begin{array}{l}2 \text { patients were on CAPD } \\
13 \text { Patients were on CCPD }\end{array}$ \\
\hline
\end{tabular}

Table 1:Baseline characteristics of patients

TIBC: Total Iron Binding Capacity; UIBC: Unsaturated Iron Binding Capacity; CAPD: Continuous Ambulatory Peritoneal Dialysis; CCPD: Continuous Cycling Peritoneal Dialysis

Univariate regression analysis was done in order to check to see if FGF-23 had influenced one of the variables. Using univariate 
Citation: Singh R, Chennasamudram SP, Sheth S, Vasylyeva TL (2014) Correlation of Fibroblast Growth Factor 23 with Markers of Inflammation and Endothelial Dysfunction in End-Stage Renal Disease and Type 2 Diabetes Patients on Peritoneal Dialysis. J Diabetes Metab 5: 371. doi:10.4172/2155-6156.1000371

Page 3 of 5

regression analysis, a model was created by using FGF-23 as a dependent variable and IL-6, PAI-1, and CD146 as single independent variables. The same univariate model was adjusted by adding three more independent variables, i.e. calcium $\times$ phosphorus product $(\mathrm{Ca} \times$ PO4 product), serum phosphate (Phos) and LDL. Multiple regression analysis was performed to analyze whether mineral metabolism significantly affected associations that were found using univariate regression.

Two-sided $p$ values $<0.05$ were considered to represent statistically significant differences. Data were analyzed using IBM SPSS version 20.0 (SPSS, Chicago, IL).

\section{Results}

Socio-demographic characteristics and basic biochemical data of 15 patients are presented in Table 1 . As expected, the study participants had elevated Phos levels. FGF-23 showed the significant and strong positive correlation with inflammatory marker IL6 $(\mathrm{r}=0.36 ; \mathrm{p}<0.05)$, and with two of the endothelial dysfunction markers: PAI-1 $(\mathrm{r}=0.21$; $\mathrm{p}<0.05)$ and CD $146(\mathrm{r}=0.29 ; \mathrm{p}<0.05)$ (Table 2). In addition, both IL-6 and IL-1 $\beta$ were positively correlated with levels of Phos, and IL-6 was positively correlated with the CaxPhos product. The same degree of positive correlation was noticed between markers of endothelial dysfunction (ET-1 and CD146) and Phos.

\begin{tabular}{|l|l|l|l|l|l|l|l|l|l|l|}
\hline Variables & FGF-23 & ET-1 & sICAM & IL6 & PAI-1 & TNFa & sVCAM & IL-1 $\beta$ & CD146 & CRP \\
\hline FGF-23 & 1.00 & 0.13 & -0.17 & $0.36^{*}$ & $0.21^{*}$ & 0.01 & -0.12 & -0.12 & $0.29^{*}$ & 0.14 \\
\hline Calcium (mg/dL) & 0.11 & $-0.26^{*}$ & 0.08 & $-0.47^{*}$ & -0.11 & 0.04 & -0.06 & -0.12 & -0.10 & $-0.27^{*}$ \\
\hline Phosphate (mg/dL) & $0.54^{*}$ & $0.49^{*}$ & -0.11 & $0.45^{*}$ & 0.11 & 0.07 & $0.20^{*}$ & $0.24^{*}$ & $0.22^{*}$ & 0.19 \\
\hline Ca×PO4 product & $0.63^{*}$ & $0.41^{*}$ & -0.09 & $0.29^{*}$ & 0.07 & 0.09 & 0.19 & 0.20 & 0.19 & 0.09 \\
\hline Triglyceride (mg/dL) & -0.23 & -0.16 & $0.48^{*}$ & 0.05 & $0.34^{*}$ & 0.11 & $-0.33^{*}$ & $0.31^{*}$ & 0.19 & -0.001 \\
\hline LDL (mg/dL) & $-0.28^{*}$ & 0.08 & 0.19 & $-0.47^{*}$ & -0.14 & -0.15 & 0.11 & 0.18 & -0.02 & -0.17 \\
\hline HDL (mg/dL) & -0.004 & $0.26^{*}$ & $-0.42^{*}$ & 0.01 & -0.16 & -0.18 & $0.53^{*}$ & -0.05 & $-0.34^{*}$ & 0.10 \\
\hline Total Cholesterol & -0.35 & 0.19 & 0.30 & -0.36 & 0.02 & -0.14 & 0.14 & 0.35 & 0.01 & -0.06 \\
\hline Intact PTH (pg/mL) & -0.02 & 0.12 & -0.05 & 0.02 & 0.01 & -0.08 & 0.02 & -0.04 & $-0.29^{*}$ & 0.03 \\
\hline
\end{tabular}

Table 2: Spearman correlation between markers of mineral metabolism and cardiovascular risks factors; FGF-23: Fibroblast Growth factor-23; ET-1: Endothelin-1; sICAM: soluble Intercellular Adhesion Molecule; IL6: Interleukin 6; PAI-1: Plasminogen Activator Iinhibitor-1; TNFa: Tumor Necrosis Factor-alpha; sVCAM: soluble Vascular Adhesion Molecule-1; IL-1 $\beta$ : Interleukin 1 $\beta$; CD 146: Cluster of Differentiation 146; CRP:C-Reactive Protein; Cax PO4product: Calcium Phosphorus product; LDL: Low-Density Lipoprotein; HDL: High-Density Lipoprotein. * $\mathrm{p}<0.05$ correlations are statistically significant. Serum concentrations of FGF-23, sICAM, PAI-1, sVCAM, and CD146 were measured in ng/mL; Serum concentrations of ET-1, IL-6, TNF $\alpha$, IL-1 $\beta$, and CRP were measured in $\mathrm{pg} / \mathrm{mL}$.

FGF-23 also had strong positive and significant correlations with Phos ( $\mathrm{r}=0.54 ; \mathrm{p}<0.05), \quad \mathrm{Ca} \times \mathrm{PO}_{4} \quad \operatorname{product}(\mathrm{r}=0.63 ; \mathrm{p}<0.05)$, and $\operatorname{LDL}(\mathrm{r}=0.28 ; \mathrm{p}<0.05)$.

The multiple regression model with all four predictors produced adjusted $\mathrm{R}^{2}=0.54,0.55,0.57 ; \mathrm{p}<0.001$, respectively for IL-6, PAI- 1 , and CD146 (Table 3). This indicated strong associations between FGF-23 andIL-6, PAI-1, and CD146.

\begin{tabular}{|l|l|l|l|l|}
\hline Variable & \multicolumn{2}{|l|}{ Univariate } & \multicolumn{2}{l|}{ Multivariate adjusted modelt } \\
\hline & $\begin{array}{l}\text { Difference } \\
(\mathbf{9 5 \%} \mathbf{C l})\end{array}$ & $\mathbf{p}$ value & $\begin{array}{l}\text { Difference } \\
\mathbf{( 9 5 \% ~ C l )}\end{array}$ & $\mathbf{p}$ value \\
\hline InFGF-23 & & & & \\
\hline InIL-6 & 0.11 & $\mathrm{p}=0.01$ & 0.54 & $\mathrm{p}<0.001$ \\
\hline InPAl-1 & 0.02 & $\mathrm{p}=0.01$ & 0.55 & $\mathrm{p}<0.001$ \\
\hline InCD 146 & 0.06 & $\mathrm{p}=0.05$ & 0.57 & $\mathrm{p}=0.05$ \\
\hline
\end{tabular}

Table 3: Univariate and multivariate linear regression analyses between inflammatory markers as dependent variables and fgf- 23 as the principal factor

CI: Confidence interval; Ln: Log-normal; FGF-23: Fibroblast Growth Factor-23; IL-6: Interleukin 6; PAI-1: Plasminogen Activator
Iinhibitor-1; CD 146: Cluster of Differentiation 146. $†$ Model adjusted for Calcium $\times$ phosphorus product: serum phosphate and low-density lipoprotein

The results were qualitatively improved in models that were further adjusted for $\mathrm{Ca} \mathrm{PO}_{4}$ product, serum Phos, and LDL (Table 3). Significant interactions were detected between FGF-23and IL-6 $(\mathrm{p}<0.001)$, PAI-1 $(\mathrm{p}<0.001)$, and CD146 in the multivariable-adjusted model (Table 3).

\section{Discussion}

In CKD and ESRD patients, high levels of FGF-23 are associated with increased morbidity and mortality rates $[10,11]$. In the past, the major risk factors for morbidity and mortality in dialysis patients were primarily linked to hypercholesterolemia, hypertension, and obesity. More recently, risk factors such as anemia, inflammation, and abnormalities in bone and mineral metabolism were incorporated [3]. These risk factors are considered to be responsible for the higher mortality rates witnessed in CKD and ESRD patients [18]. However, without in-depth knowledge of the pathophysiologic mechanisms, it is difficult to identify efficient targets for therapeutic interventions. Over the past few years, FGF-23 has been recognized as a predictor of a poor prognosis in ESRD patients [18]. In the present study, correlation and association of FGF-23 levels with IL-6, PAI-1, CD 146, and some other inflammatory, endothelial dysfunction factors, and mineral 
Citation: Singh R, Chennasamudram SP, Sheth S, Vasylyeva TL (2014) Correlation of Fibroblast Growth Factor 23 with Markers of Inflammation and Endothelial Dysfunction in End-Stage Renal Disease and Type 2 Diabetes Patients on Peritoneal Dialysis. J Diabetes Metab 5: 371. doi:10.4172/2155-6156.1000371

Page 4 of 5

metabolites in ESRD patients with T2DM were investigated. This association was studied separately from other inflammationpromoting factors such as total cholesterol and triglyceride.

Several studies have confirmed that FGF-23 might be responsible for mortality, Left Ventricular Hypertrophy (LVH), and the progression of CKD, independent of serum phosphate levels [19-21]. Findings in this study further corroborates the observations of previous studies that there is a strong correlation in the levels of FGF-23 and interleukins in ESRD patients undergoing hemodialysis [15] and in CKD patients not yet on dialysis [2].

In our study, a unique population of patients with the highest mortality rate (T2DM patients with ESRD on PD) demonstrated correlations between FGF-23 level and acute phase response markers such as IL-6, and endothelial dysfunction markers, in particular PAI-1 and CD 146.

Faulet al. Recently demonstrated a direct role for elevated FGF-23 in the pathogenesis of LVH and suggested a new mechanism to explain the high rates of $\mathrm{LVH}$ in patients with CKD. They reported that FGF-23 directly promoted pathological hypertrophy of isolated cardiomyocytes and that mice developed $\mathrm{LVH}$ after intra-ventricular or intravenous injection of FGF-23 [22]. In our study, FGF-23 did not significantly correlate with the endothelial dysfunction markers, ET-1, sICAM, or sVCAM. Similar findings were observed by Almorth et al. in ESRD patients on HD [23]. Concurrently, we found that the markers PAI-1 and CD 146 and $\mathrm{Ca} \times \mathrm{PO}_{4}$ product were more sensitive than ET-1, sICAM, or sVCAM in the presence of elevated FGF-23. As we found in our study, sICAM and sVCAM were more tightly associated with lipid metabolism than to the mineral misbalance stage, although ET-1 and sVCAM showed some positive correlations with Phos. These interesting finding require further research to understand the relationships between these major players in cardiovascular health.

FGF-23 has been reported to reduce levels of circulatory 1,25dihydroxycholecalciferol $(1,25 \mathrm{D})$ by inhibition of renal 1-hydroxylase and stimulation of 24-hydroxylase, ultimately enhancing the rate of $1,25 \mathrm{D}$ decay $[24,25]$. Since reduced $1,25 \mathrm{D}$ levels are associated with higher levels of pro-inflammatory markers such as IL-6 and ET-1 [26], it is possible that FGF-23 mediates increase of some endothelial inflammation and endothelial dysfunction markers, acting through $1,25 \mathrm{D}$. This assumption of indirect action requires further investigation.

Determining the mechanisms involved in cross-sectional interrelationships between FGF-23 and different inflammatory markers area complicated task. It is possible that FGF-23 acts directly to induce inflammation. Mendoza et al. reported that FGF-23 induces signaling in cells that do not express klotho, via activation of FGF receptors [27], which are present in adipose tissue [28]. Thus, it is possible that FGF-23 could induce expression of fat-derived cytokines, such as IL-6, PAI-1, and CD $146[29,30]$ through klotho-independent mechanisms. Chitaliaet al. Findings were similar to our results in that FGF-23 was associated with increased interleukins [2].

In addition, observations from ourstudy concur with earlier studies that reported an association between levels of serum phosphate and inflammatory markers in ESRD patients $[27,28]$. We observed that serum phosphate levels are also correlated with the inflammatory and endothelial dysfunction markers ET-1, IL-6, CD146, IL-1 $\beta$, and VCAM. These findings underline the critical role played by serum phosphate in the occurrence of inflammation and micro-vascular problems in ESRD patients.

\section{Conclusion}

Our results indicate that FGF-23 impacts the cardiovascular health of T2DM patients on PD through mechanisms, which are independent from phosphate levels and linked directly to inflammation and endothelial dysfunction. Further studies are necessary to explainthe precise mechanisms through which FGF-23 might impact morbidity and mortality of ESRD patientswith T2DM on PD. Studies of FGF-23 pathogenicity may also help in the identification of new therapeutic targets and the development of new therapeutic methods.

\section{Acknowledgement}

We appreciate Sanofi, Inc., Cambridge, MA for sponsorship of our research, Candace Myers $\mathrm{PhD}$ for reviewing the manuscript, and Grant Weinheimer for technical support. We appreciate Dr. Georges M. Maliha and Dr.Giron Milton for allowing us to enroll patients from their practice.

\section{References}

1. Kovesdy CP, Quarles LD (2013) The role of fibroblast growth factor-23 in cardiorenal syndrome. Nephron Clin Pract 123: 194-201.

2. Chitalia N, Afzali B, Edozie F, Manghat P, Wierzbicki AS, et al. (2012) Serum FGF-23 is linked with increased inflammatory cytokines in CKD stage 3-5. Is this the explanation for its adverse CV effects? Abstract Am Soc Nephrol.

3. Gutiérrez OM, Mannstadt $\mathrm{M}$, Isakova $\mathrm{T}$, Rauh-Hain JA, Tamez $\mathrm{H}$, et al. (2008) Fibroblast growth factor 23 and mortality among patients undergoing hemodialysis. N Engl J Med 359: 584-592.

4. Oliveira RB, Cancela AL, Graciolli FG, Dos Reis LM, Draibe SA, et al. (2010) Early control of PTH and FGF23 in normophosphatemic CKD patients: a new target in CKD-MBD therapy? Clin J Am Soc Nephrol 5: 286-291.

5. Yilmaz MI, Sonmez A, Saglam M, Yaman H, Kilic S, et al. (2012) Comparison of calcium acetate and sevelamer on vascular function and fibroblast growth factor 23 in CKD patients: a randomized clinical trial. Am J Kidney Dis 59: 177-185.

6. Yilmaz MI, Sonmez A, Saglam M, Yaman H, Kilic S, et al. (2010) FGF-23 and vascular dysfunction in patients with stage 3 and 4 chronic kidney disease. Kidney Int 78: 679-685.

7. EVOLVE Trial Investigators, Chertow GM, Block GA, Correa-Rotter R, Drüeke TB, et al. (2012) Effect of cinacalcet on cardiovascular disease in patients undergoing dialysis. N Engl J Med 367: 2482-2494.

8. Abu-Alfa AK (2003) The ADEMEX study: Expanding the boundaries of peritoneal dialysis adequacy beyond small solute clearances. Dialysis \& Transplantation 32: 115.

9. Ma KW, Greene EL, Raij L (1992) Cardiovascular risk factors in chronic renal failure and hemodialysis populations. Am J Kidney Dis 19: 505-513.

10. Yeun JY, Levine RA, Mantadilok V, Kaysen GA (2000) C-Reactive protein predicts all-cause and cardiovascular mortality in hemodialysis patients. Am J Kidney Dis 35: 469-476.

11. LameireN, Bernaert P, Lambert MC, Vijt D (1994) Cardiovascular RiskFactors and Their Management in Patients on Continuous Ambulatory Peritoneal-Dialysis. Kidney Int Suppl: S31-S38.

12. Ducloux D, Bresson-Vautrin C, Kribs M, Abdelfatah A, Chalopin JM (2002) C-reactive protein and cardiovascular disease in peritoneal dialysis patients. Kidney Int 62: 1417-1422.

13. Bergstrom J HO, Lindholm B, Qureshi AR (1995) Elevated serum Creactive protein is a stronger predictor of increased mortality and low serum albumin in heamodialysis patients. J Am Soc Nephrol (abstract) 6: 573.

14. Koenig W, Sund M, Fröhlich M, Fischer HG, Löwel H, et al. (1999) CReactive protein, a sensitive marker of inflammation, predicts future risk 
Citation: Singh R, Chennasamudram SP, Sheth S, Vasylyeva TL (2014) Correlation of Fibroblast Growth Factor 23 with Markers of Inflammation and Endothelial Dysfunction in End-Stage Renal Disease and Type 2 Diabetes Patients on Peritoneal Dialysis. J Diabetes Metab 5: 371. doi:10.4172/2155-6156.1000371

Page 5 of 5

of coronary heart disease in initially healthy middle-aged men: results from the MONICA (Monitoring Trends and Determinants in Cardiovascular Disease) Augsburg Cohort Study, 1984 to 1992. Circulation 99: 237-242.

15. Marinelli A, Orlandi L, Stivali G (2011) C-reactive protein levels are associated with arterial media calcification in nondiabetic patients with end-stage renal disease on long-term hemodialysis. Clin Nephrol 76: 425-434.

16. Brandenburg VM, Schlieper G, Heussen N, Holzmann S, Busch B, et al. (2010) Serological cardiovascular and mortality risk predictors in dialysis patients receiving sevelamer: a prospective study. Nephrol Dial Transplant 25: 2672-2679.

17. Paulie S, Perlmann H, Perlmann P (2001) Enzyme-linked Immunosorbent Assay. eLS: John Wiley \& Sons, Ltd.

18. Kovesdy CP, Quarles LD (2013) Fibroblast growth factor-23: what we know, what we don't know, and what we need to know. Nephrol Dial Transplant 28: 2228-2236.

19. Wang AY (2011) Vascular and valvular calcification in chronic peritoneal dialysis patients. Int J Nephrol 2011: 198045

20. Wang AY, Ho SS, Wang M, Liu EK, Ho S, et al. (2005) Cardiac valvular calcification as a marker of atherosclerosis and arterial calcification in end-stage renal disease. Arch Intern Med 165: 327-332.

21. Wang AY (2007) Cardiovascular risk factors in peritoneal dialysis patients revisited. Perit Dial Int 27: S223-227.

22. Faul C, Amaral AP, Oskouei B, Hu MC, Sloan A, et al. (2011) FGF23 induces left ventricular hypertrophy. J Clin Invest 121: 4393-4408.

23. Almroth G, Lönn J, Uhlin F, Nayeri F, Brudin L, et al. (2013) Fibroblast Growth Factor 23, hepatocyte growth factor, interleukin 6, high senstive creative protein and soluble urokinase plasminogen activator receptors: Inflammation markers in chronic hemodialysis patients? Scand J Immunol 78: 285-290.

24. Hasegawa H, Nagano N, Urakawa I, Yamazaki Y, Iijima K, et al. (2010) Direct evidence for a causative role of FGF23 in the abnormal renal phosphate handling and vitamin D metabolism in rats with early-stage chronic kidney disease. Kidney Int 78: 975-980.

25. Shimada T, Hasegawa H, Yamazaki Y, Muto T, Hino R, et al. (2004) FGF-23 is a potent regulator of vitamin D metabolism and phosphate homeostasis. J Bone Miner Res 19: 429-435.

26. Isakova T, Gutiérrez OM, Patel NM, Andress DL, Wolf M, et al. (2011) Vitamin D deficiency, inflammation, and albuminuria in chronic kidney disease: complex interactions. J Ren Nutr 21: 295-302.

27. Munoz Mendoza J, Isakova T, Ricardo AC, Xie H, Navaneethan SD, et al. (2012) Fibroblast growth factor 23 and Inflammation in CKD. Clin J Am SocNephrol 7: 1155-1162.

28. Kurosu H, Choi M, Ogawa Y, Dickson AS, Goetz R, et al. (2007) Tissuespecific expression of betaKlotho and fibroblast growth factor (FGF) receptor isoforms determines metabolic activity of FGF19 and FGF21. J Biol Chem 282: 26687-26695.

29. Axelsson J, Rashid Qureshi A, Suliman ME, Honda H, Pecoits-Filho R, et al. (2004) Truncal fat mass as a contributor to inflammation in end-stage renal disease. Am J Clin Nutr 80: 1222-1229.

30. Wisse BE (2004) The inflammatory syndrome: The role of adipose tissue cytokines in metabolic disorders linked to obesity. J Am Soc Nephrol 15: 2792-2800. 